\title{
Molecular characterization of the Jatropha curcas JCR1MYB1 gene encoding a putative R1-MYB transcription factor
}

\author{
Hui-Liang Li, Dong Guo and Shi-Qing Peng \\ Key Laboratory of Biology and Genetic Resources of Tropical Crops, Ministry of Agriculture, \\ Institute of Tropical Bioscience and Biotechnology, Chinese Academy of Tropical Agricultural Sciences, \\ Haikou, China.
}

\begin{abstract}
The cDNA encoding the R1-MYB transcription factor, designated as JcR1MYB1, was isolated from Jatropha curcas using rapid amplification of cDNA ends. JcR1MYB1 contains a $951 \mathrm{bp}$ open reading frame that encodes 316 amino acids. The deduced JcR1MYB1 protein was predicted to possess the conserved, 56-amino acid-long DNA-binding domain, which consists of a single helix-turn-helix module and usually occurs in R1-MYBs. JcR1MYB1 is a member of the R1-MYB transcription factor subfamily. A subcellular localization study confirmed the nuclear localization of JcR1MYB1. Expression analysis showed that $J c R 1 M Y B 1$ transcripts accumulated in various examined tissues, with high expression levels in the root and low levels in the stem. JcR1MYB1 transcription was up-regulated by polyethylene glycol, $\mathrm{NaCl}$, and cold treatments, as well as by abscisic acid, jasmonic acid, and ethylene treatment. Analysis of transgenic tobacco plants over-expressing JcR1MYB1 indicates an inportant function for this gene in salt stress.
\end{abstract}

Keywords: abiotic stress,gene expression, Jatropha curcas, R1-MYB transcription factor.

Received: January 17, 2014; Accepted: May 16, 2014.

\section{Introduction}

MYB proteins constitute a large family of transcription factors (TFs), which is functionally diverse and is represented in all eukaryotes (Dubos et al., 2010). MYB proteins are characterized by a highly conserved MYB DNA-binding domain with up to three imperfect repeats, each comprised of an approximately 50-amino acid-long, helix-turn-helix structure (Jin and Martin, 1999). Based on the numbers of adjacent imperfect repeats (R1, R2, and R3) in the DNA-binding domain, MYB TFs are classified into four subfamilies, namely, R2R3-MYB, R1-MYB, R3MYB, and R4-MYB factors (Dubos et al., 2010). Members of the MYB family have important functions in plant development, metabolism, and stress responses (Allan et al., 2008; Dubos et al., 2010). Most plant MYB TFs belong to the R2R3-type (Du et al., 2009). Compared with R2R3type MYB TFs, few reports exist on functional studies of MYB TFs with a single helix-turn-helix structure in plants. Potato MybSt1 is the first reported MYB transcription factor possessing such a single module in plants (Wang et al., 1997). In Arabidopsis, CIRCADIAN CLOCK ASSOCIATED (CCA1) is a MYB protein with only a he-

Send correspondence to Shi-Qing Peng. Key Laboratory of Biology and Genetic Resources of Tropical Crops, Ministry of Agriculture, Institute of Tropical Bioscience and Biotechnology, Chinese Academy of Tropical Agricultural Sciences, \#4 Xueyuan Rd., Haikou 571101, China. E-mail: shqpeng@163.com. lix-turn-helix motif and functions as a specific activator of phytochrome signal transduction (Wang et al., 1997). Constitutive expression of CCA1 results in longer hypocotyls and substantially delayed flowering (Wang and Tobin, 1998). In rice, ANTHER INDEHISCENCE1 (AID1) is closely related to other single MYB-like domain TFs in plants. AID1 was identified in a genetic screen as playing a role in partial to complete spikelet sterility (Zhu et al., 2004). StMYB1R-1 was recently identified as a putative stress-response gene. Over-expression of StMYB1R-1 in potato plants improved plant tolerance to drought stress (Shin et al., 2011). Growing evidence suggests that R1MYB TFs have diverse functions in plant growth, development, and stress responses (Lee and Schiefelbein, 1999; Kuno et al., 2003; Rubio-Somoza et al., 2006; Jia et al., 2009; Cheng et al., 2013).

Jatropha curcas L. (physic nut) is a woody oil plant that is found in tropical and subtropical countries. Physic nut produces oil from the seeds, which can be combusted as fuel without prior refining (Openshaw, 2000; Fairless, 2007; Sato et al., 2011). A draft of the J. curcas genome sequence has recently been reported (Sato et al., 2011). The genome-wide MYB genes were identified and described in the physic nut (data not shown). In this work, one member of the physic nut R1-MYB family, designated as $J_{C R} 1 M Y B 1$, was investigated for its expression pattern. 
Moreover, the salt tolerance of transgenic JcRIMYBI tobacco was evaluated.

\section{Materials and Methods}

Plant materials, plant hormones, and stress treatments

Mature J. curcas seeds were collected from the South China Botanical Garden, Chinese Academy of Sciences, Guangdong Province, China. The seeds were surface sterilized in $70 \%$ ethanol for $10 \mathrm{~min}$, then in $10 \% \mathrm{NaClO}$ for $10 \mathrm{~min}$. The seeds were rinsed four times with sterile distilled water. The cotyledons were then removed from the seeds and were placed in $100 \mathrm{~mL}$ flasks containing $40 \mathrm{~mL}$ of Murashige and Skoog (MS) medium and 0.6\% (w/v) agar at $\mathrm{pH} 5.8$. After $3 \mathrm{~d}$, the rooted cotyledons were transferred into pots with 1:1 (v/v) vermiculite and peat medium and then incubated at $28{ }^{\circ} \mathrm{C}$ with a $16 \mathrm{~h}$ light $/ 8 \mathrm{~h}$ dark photoperiod for three weeks. Three-week-old light-grown intact plants (with two to three leaves) were used for polymerase chain reaction (PCR) analysis. Chemical treatment was performed as follows: a solution of $200 \mathrm{mM} \mathrm{NaCl}$, $20 \%$ polyethylene glycol (PEG), $100 \mathrm{mM} \mathrm{ABA}, 50 \mathrm{mM}$ ethephon (ET), and $100 \mathrm{mM}$ jasmonic acid (JA) were applied to the surface of solid MS agar medium of the threeweek-old seedlings. For cold treatment, the seedlings incubated at $4{ }^{\circ} \mathrm{C}$ under continuous light for $1 \mathrm{~d}$. After each treatment, sample seedlings were harvested and immediately frozen in liquid nitrogen until use for real-time quantitative PCR (RT-qPCR).

\section{Isolation of RNA}

Total RNA was extracted according to the method by Chang et al. (1993). The quality and concentration of the extracted RNA was verified using agarose gel electrophoresis and was measured with a spectrophotometer (DU-70, Beckman, Fullerton, CA).

\section{Cloning of JcR1MYB1}

Rapid amplification of cDNA ends (RACE) was used to obtain the DNA sequence encoding a putative R1-MYB $\mathrm{TF}$ based on the genome sequence at http://www.kazusa.or.jp/jatropha/ (Sato et al., 2011). Moreover, 3'- and 5'-RACE were conducted using the double-stranded cDNA from J. curcas as a template. The primers used for the 3' RACE and the 5' RACE were designed based on the sequence (Table 1). The amplified product was purified (Tiangen, China) and cloned into the pGEM-T easy vector (Promega, USA) and then sequenced. The sequences were compared with those in the NCBI database using the basic local alignment search tool (BLAST). Based on the 5' and 3' end cDNA sequences, primers were designed to enable amplification of the entire JCRIMYB1. The amplified products were purified (Tiangen, China) and
Table 1 - Primer sequences (Nucleotide sequences from 5'to 3').

\begin{tabular}{ll}
\hline 3'RACE-PCR primers & \\
3MYB11 & GAATGCCAAGGAATGCTCCCAGTCGAT \\
3MYB12 & TTGCCAGATCGGATTGGTGAATGCTCC \\
5' RACE-PCR primers & \\
5MYB11 & GGATTGCTTTCCCAGCCTGTATTTCTG \\
5MYB12 & CCTTTACTCCCATTGTCCTCATAATCG \\
Real time PCR primers & \\
RF1 & AGACCAAGGCTTGCATTTGGT \\
RF2 & TAAATGTCTTTGCCACTCATCC \\
JCACT specific primers & \\
AF & CAGTGGTCGACAACTGGTAT \\
AR & TCCTCCAATCCAGACACTGT \\
\hline
\end{tabular}

cloned into the pGEM-T easy vector (Promega, USA) and then sequenced.

\section{Subcellular localization of JcR1MYB1}

The $J C R 1 M Y B 1$ coding region was fused in frame to the 5 ' terminus of the gene that encodes green fluorescent protein (GFP) under the control of the CaMV35S promoter in the pCAMBIA1302 vector. The resulting JcR1MYB1GFP fusion construct was used for transient expression in onion epidermal cells. The location of the introduced gene in the onion cells was observed under an adaptive optics fluorescence microscope with ultraviolet excitation filter.

\section{Expression analysis of $J C R 1 M Y B 1$}

RT-qPCR was conducted with primers (Table 1). RT-qPCR was performed using the fluorescent dye SYBR-Green (Takara, Dalian, China) and the BIO-RAD CFX96 real-time PCR system (Bio-Rad, USA) using the following protocol: denaturation at $95{ }^{\circ} \mathrm{C}$ for $30 \mathrm{~s}$, and amplification at $94^{\circ} \mathrm{C}$ for $5 \mathrm{~s}$, at $60^{\circ} \mathrm{C}$ for $20 \mathrm{~s}$, and at $72{ }^{\circ} \mathrm{C}$ for $20 \mathrm{~s}$. Three biological replicates were run, and triplicate quantitative assays were performed for each biological replicate. The actin gene from J. curcas was amplified as internal control. The relative abundance of transcripts was calculated according to the Bio-Rad CFX Manager (Version 1.5.534) of BIO-RAD CFX96.

\section{Plasmid construction and plant transformation}

The JCR1MYB1 coding region was cloned into pBI121, which contains the CaMV 35S promoter fragment. Transgenic tobacco plants were generated by transforming the pBI 121-JcR1MYB1 constructs into leaves of 6- to 8-week-old tobacco (Nicotiana tabacum cultivar Samsun $\mathrm{NN}$ ) by means of the Agrobacterium tumefaciens-mediated leaf disc method. The plant growth conditions, transformation, selection of transformants, and determination of genotyping the $\mathrm{T}_{2}$ generation were performed as described by Pontier et al. (1994). JcR1MYB1 expression in transgenic 
lines was tested by reverse transcription PCR (RT-PCR) assays, using total RNA from transgenic plants amplified with $J C R 1 M Y B 1$ specific primers (Table 1). NtACT used as internal control parallel was amplified with $N t A C T$ specific primers AF (5'-CAGTGGCCGTACAACAGGTAT-3') and AR (5'-ATCCTCCAAT CCAGACACTGT-3'). PCR assays consisted of a $5 \mathrm{~min}$ preheat at $95^{\circ} \mathrm{C}$ and 22 cycles of $30 \mathrm{~s}$ at $95^{\circ} \mathrm{C}, 30 \mathrm{~s}$ at $55^{\circ} \mathrm{C}$, and $45 \mathrm{~s}$ at $72^{\circ} \mathrm{C}$, followed by a 10 min final extension at $72{ }^{\circ} \mathrm{C}$. The PCR products were analyzed through agarose gel electrophoresis with ethidium bromide staining.

\section{Tolerance of transgenic tobacco plants to salt stress}

Seeds were surface sterilized in $70 \%$ ethanol for $10 \mathrm{~min}$, followed by $10 \% \mathrm{NaClO}$ for $10 \mathrm{~min}$. After rinsing four times with sterile distilled water the seeds were placed in solid Murashige and Skoog (MS) medium containing $200 \mathrm{mM} \mathrm{NaCl}$. Seed germination rate was analyzed after 6 days.

For the detached leaf disc $\mathrm{NaCl}$ stress treatments, $10 \mathrm{~mm}$ diameter tobacco leaf discs from four-week-old seedlings of the $\mathrm{T}_{2}$ generation $J_{C} R 1 M Y B 1$ transgenic plants were soaked in $150 \mathrm{mM} \mathrm{NaCl}$ for 4 days. The control plants were treated with $\mathrm{H}_{2} \mathrm{O}$ under the same conditions. All plants were treated and incubated under the same conditions at $24{ }^{\circ} \mathrm{C} \pm 2{ }^{\circ} \mathrm{C}$ and $65 \% \pm 5 \%$ relative humidity during the experiment.

\section{Expression analysis of $J c R 1 M Y B 1$ in transgenic tobacco plants}

The seeds were surface sterilized as described above and, after rinsing, placed in solid Murashige and Skoog (MS) medium. Three-week-old light-grown intact plants were used for RT-qPCR analysis. Chemical treatment was performed as follows: $100 \mathrm{mM}$ absciscic acid (ABA), $50 \mathrm{mM} \mathrm{ET}$, and $100 \mathrm{mM} \mathrm{JA}$ were applied to the surface of the solid MS agar medium of the three-week-old seedlings. After $2 \mathrm{~h}$ in each treatment, sample seedlings were harvested and immediately frozen in liquid nitrogen until RTqPCR.

\section{Results}

\section{Characterization of $J c R 1 M Y B 1$}

The full-length cDNA that encodes a putative R1MYB TF, designated as JcR1MYB1 (GenBank accession no. KF809956), was cloned via RACE. The 1283 bp fulllength cDNA contained a 951 bp open reading frame with a

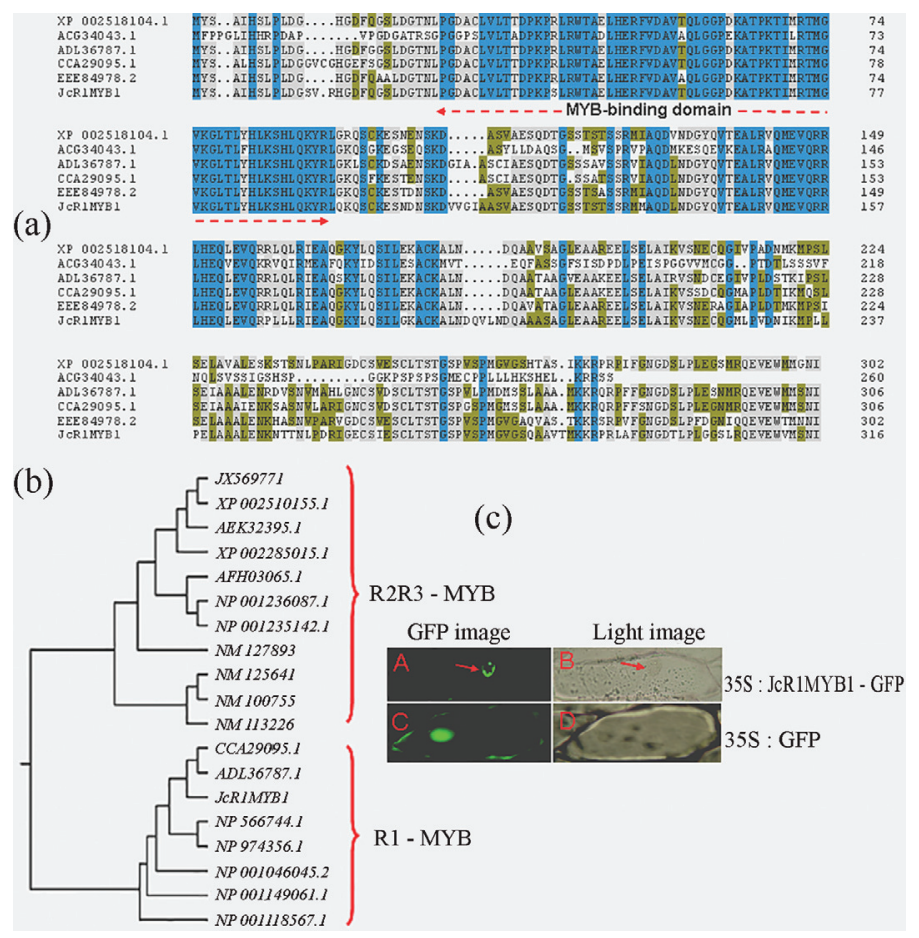

Figure 1 - Characterization of $J c R 1 M Y B 1$. (A) Amino acid sequence alignment of JcR1MYB1R1 and other single MYB-like domain proteins. Accession numbers are as follows: Ricinus communis, XP005518104.1; Zea mays, ACG34043.1; Malus domestica, ADL36787.1; Rosa rugosa, CCA29095.1; Populus trichocarpa, EEE84978.2. Identical amino acid residues are colored. (B) Phylogenetic analysis of JcR1MYB1. The phylogenic tree was constructed with the DNAMAN program using the deduced amino acid sequence. Accession numbers are as follows: Arabidopsis thaliana, NP_566744.1; NP_974356.1; NP_001118567.1; NM_127893;NM_100755; NM_113226; Oryza sativa, NP_001046045.2; Zea mays, NP_001149061.1; Jatropha curcas, JX569771.1; Ricinus communis,XP_002510155.1; Citrus sinensis, AEK32395.1; Vitis vinifera, XP_002285015.1; Epimedium sagittatum, AFH03065.1; Glycine max, NP_001238087.1; NP_001235142.1; Malus domestica, ADL36787.1; Rosa rugosa, CCA29095.1. (C) Nuclear localization of the JcR1MYB1 -GFP fusion protein in onion epidermal cells. The GFP fluorescence images (GFP image and light image) of onion epidermal cells were compared to show the subcellular localization of 35S-JcR1MYB1-GFP (A) and of 35S-GFP (C). 
192 bp 3' UTR downstream from the stop codon and a 137 bp 5' UTR upstream of the start codon. BLAST analysis showed that the JCR1MYB1 DNA sequence was identical to that of the corresponding full-length cDNA, suggesting that no intron exists in $J_{C} R 1 M Y B 1$.

A search for potential motifs identified the conserved, 56-amino acid-long DNA-binding domain, which consists of a single helix-turn-helix module that usually occurs in R1-MYBs. The sequence of the R1 DNA-binding domain of JcR1MYB1 showed $82 \%$ to $98 \%$ identity with R1MYB from other plants. However, these proteins shared minimal homology outside this domain (Figure 1A). JcR1MYB1 amino acid sequences with R1-MYB and R2R3-MYB from different species were compared, and the result of the phylogenetic tree analysis revealed that JcR1MYB was clearly clustered with R1-MYB of other plants (Figure 1B), which implies that JCR1MYB1 may code for a typical R1-MYB protein. The subcellular localization of JcR1MYB1 indicates that JcR1MYB1 localizes in the nucleus (Figure 1C), and that the properties of the JcR1MYB1 define it as a TF.

\section{Differential JcR1MYB1 expression in different organs}

Total RNA was isolated from the leaves, stems, and roots of $J$. curcas seedlings and was subjected to RT-qPCR to analyze the $J_{C} R 1 M Y B 1$ transcription pattern. $J_{C} R 1 M Y B 1$ was shown to be constitutively expressed in all tested tissues at different levels. The highest transcript levels were seen in stems, followed by the roots and leaves (Figure 2A).

\section{Effects of plant hormones and stress on JcR1MYB1 expression}

The JcR1MYB 1 mRNA accumulation profile was determined under various abiotic stresses by RT-qPCR to determine whether $J_{C} R 1 M Y B 1$ expression is regulated by multiple factors. Four-week-old intact $J$. curcas seedlings were treated with various chemical reagents for various durations, and the transcript levels were monitored at each time point. JCR1MYB1 expression increased within $0.5 \mathrm{~h}$, reached its maximum at $2 \mathrm{~h}$, and subsequently decreased under ethylene (ET), abscisic acid (ABA), and jasmonic acid (JA) treatment (Figure 2B). JcR1MYB1 expression increased within $0.5 \mathrm{~h}$ under $\mathrm{NaCl}$, PEG, and cold treatments. However under the $\mathrm{NaCl}$ treatment, JCR1MYBI expression increased within $2 \mathrm{~h}$ and then subsequently decreased (Figure $2 \mathrm{C})$.

Phenotypes of transgenic plants under PEG and salt stresses

JCR1MYB1 was overexpressed under the control of the CaMV 35S promoter in tobacco plants. The transgenic tobacco plants that harbored the JCRIMYB1 gene were selected using RT-PCR (Figure 3A). PCR detection of the $\mathrm{T}_{0}-\mathrm{T}_{2}$ transgenic lines showed that $J_{c} R 1 M Y B 1$ was stably inherited. The transcription of $J_{C R} 1 M Y B 1$ in $\mathrm{T}_{2}$ transgenic lines was detected by RT-PCR. JCRIMYBI was constitutively expressed in all transgenic lines, and these had higher transcript levels compared with WT (transformant host) plants (data not shown).

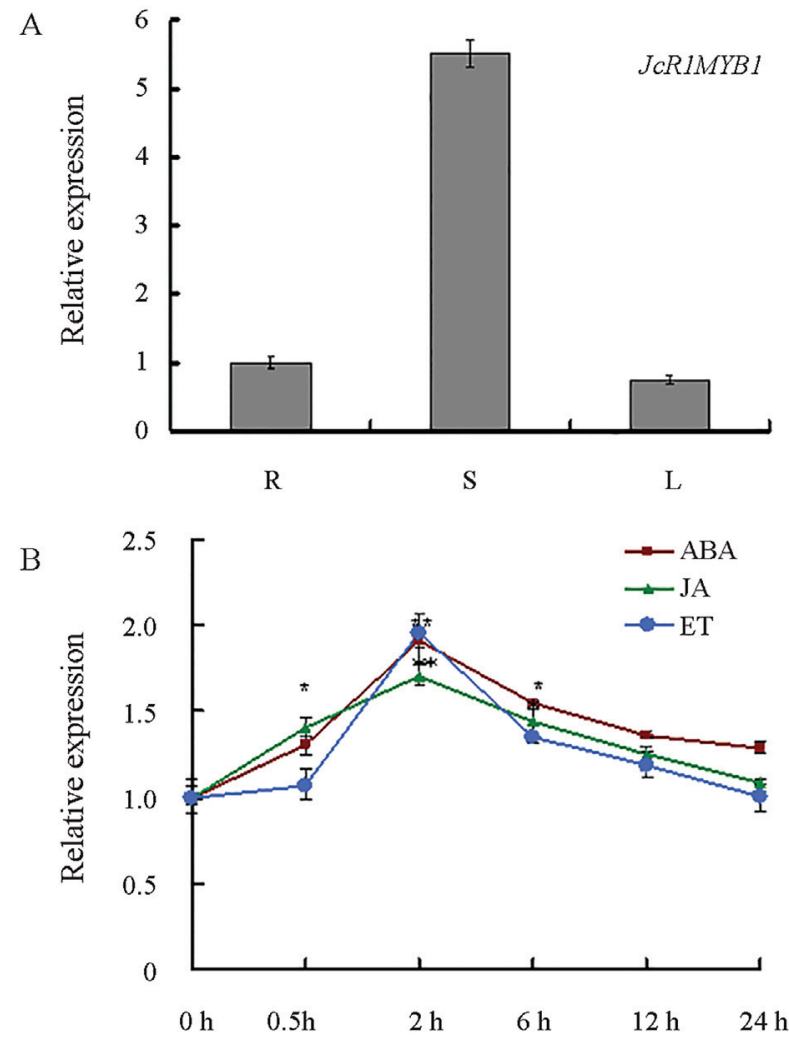

$\mathrm{C}$

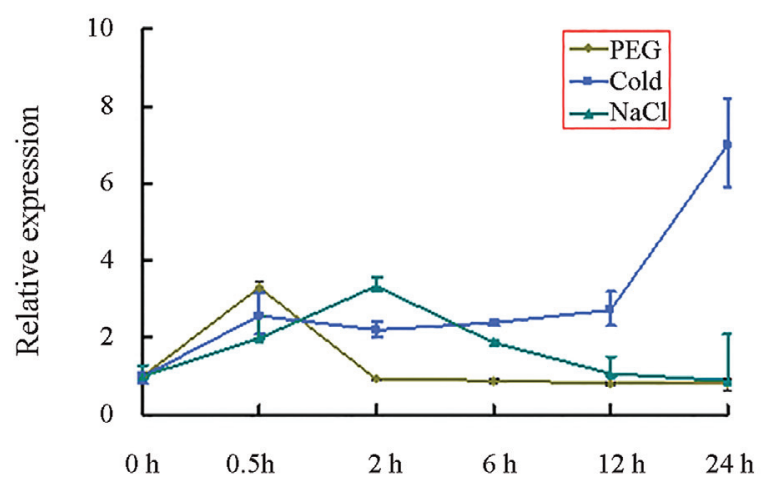

Figure 2 - Expression of $J_{C} R 1 M Y B 1$. (A) $J_{C} R 1 M Y B 1$ expression in the roots (R), stems (S), and leaves (L) of $J$. curcas seedlings. Relative transcript abundances of $J C R I M Y B 1$ were examined using RT-qPCR. Genespecific primers for $J_{C R} 1 M Y B 1$ and $J_{C} A C T$ (internal control) were used. Each point represents the mean of three replicates. Bars indicate standard errors $( \pm \mathrm{SE})$. The $\mathrm{Y}$-axis is the scale of the relative transcript abundance level. The X-axis refers to the tissues of $J$. curcas. (B) JcRIMYBI transcription patterns induced by JA, ET, and ABA treatments. (C) JcR1MYBI transcription patterns induced by $\mathrm{PEG}$, cold, and $\mathrm{NaCl}$ treatments. Relative transcript abundances of $J_{C} R I M Y B 1$ were examined by RT-qPCR. Gene-specific primers for $J_{C} R 1 M Y B 1$ and $J C A C T$ (internal control) were used. Each point represents the mean of three replicates. Bars indicate standard errors $( \pm \mathrm{SE})$. The Y-axis refers to the scale of the relative transcript abundance level. The $\mathrm{X}$-axis shows the time elapsed after the treatment. 


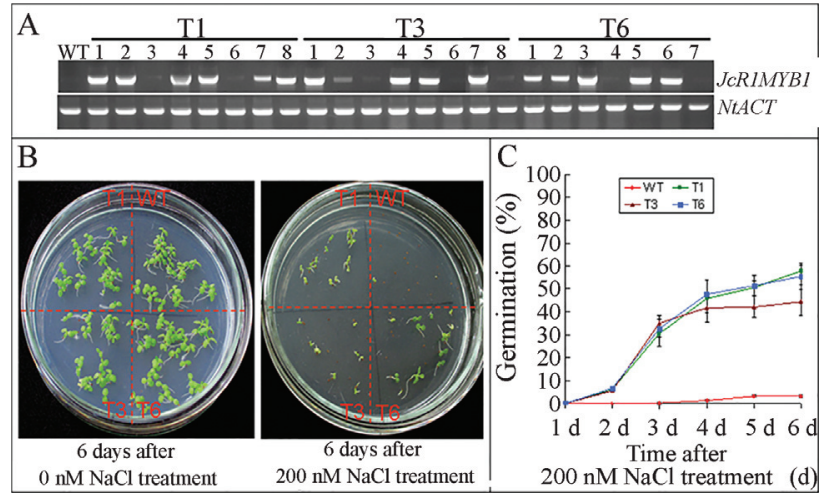

Figure 3 - Characterization of transgenic tobacco plants. (A) Molecular identification of $J c R 1 M Y B 1$ in $\mathrm{T}_{2}$ transgenic plants (T1, T3, and $\mathrm{T} 6$ lines) by RT-PCR. (B) The seed germination of WT and transgenic plants (T1, $\mathrm{T} 3$, and $\mathrm{T} 6$ lines) on MS containing $200 \mathrm{mM} \mathrm{NaCl}$. (C) The germination rate of WT and transgenic plants (T1, T3, and T6 lines) on MS containing $200 \mathrm{mM} \mathrm{NaCl}$

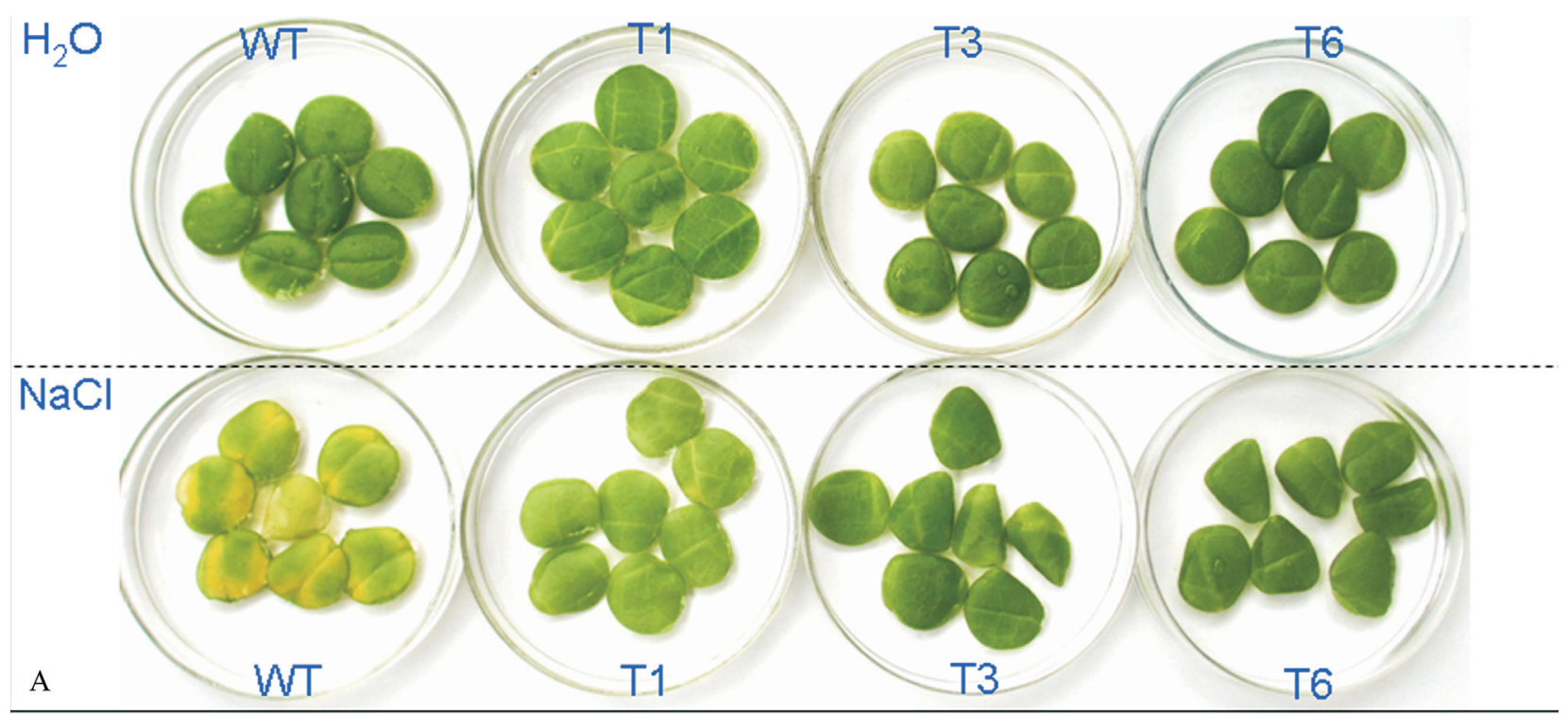

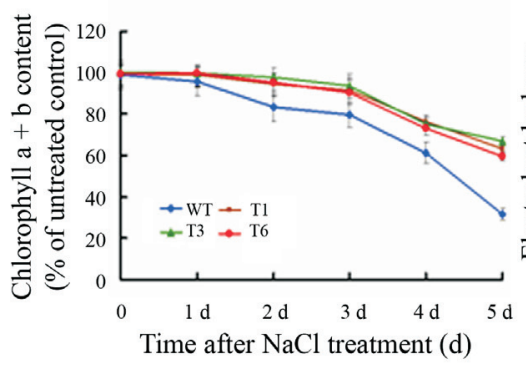

B

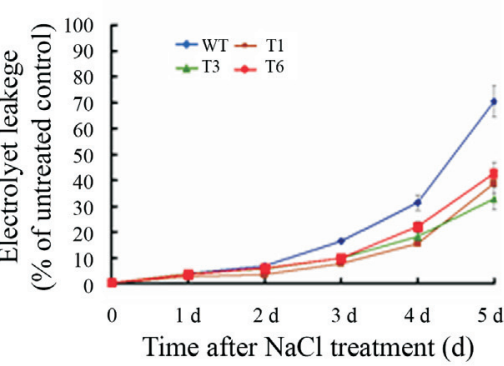

$\mathrm{C}$
The seeds and leaf discs from the $\mathrm{T}_{2}$ transgenic tobacco lines were subjected to salt stress to evaluate the response of the $J_{C} R 1 M Y B 1$ transgenic plants. The seed germination rate of $J c R 1 M Y B 1$ transgenic plants was significantly higher than that of WT on MS containing $200 \mathrm{mM} \mathrm{NaCl}$ (Figure 3B). After treatment, the leaf discs from $J_{C R} 1 M Y B 1$ transgenic plants exhibited enhanced salt tolerance relative to the WT (Figure 4A). Concomitantly, alterations in chlorophyll content and ion leakage of the leaves under $\mathrm{NaCl}$ treatment were also evaluated as reliable indices of photosynthetis and cell membrane damage under $\mathrm{NaCl}$ treatment. As shown in Figure 4B, chlorophyll contents are significantly lower in WT than in the three transgenic lines. The ion leakage in the WT plants is significantly higher than in the three transgenic lines (Figure 4C). These results indicate that $J_{C} R 1 M Y B 1$ over-expression enhanced tolerance to salt stress. $J_{C} R 1 M Y B 1$ expression in the

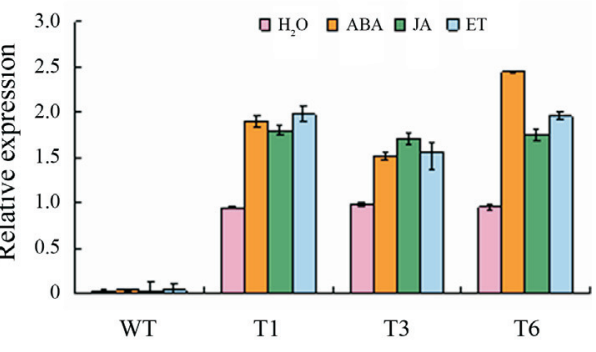

$\mathrm{D}$

Figure 4 - JCR1MYB1 transgenic tobacco phenotypes in response to salt. (A) Phenotype of leaf discs from WT and transgenic plants (T1, T3, and T6 lines) after treatment with $200 \mathrm{mM} \mathrm{NaCl}$ for $5 \mathrm{~d}$. Detached leaves from WT controls were treated with water under the same conditions. (B) Chlorophyll content of leaf discs from WT and transgenic plants (T1, T3, and T3 lines) after treatment with $200 \mathrm{mM} \mathrm{NaCl}$ for $5 \mathrm{~d}$. Error bars show standard deviations for three independent replicates. (C) Electrolyte leakage of leaf discs from WT and transgenic plants (T1, T3, and T3 lines) after treatment with $200 \mathrm{mM} \mathrm{NaCl}$ for 5 d. Error bars show standard deviations for three independent replicates. (D). Expression of $J_{c} R 1 M Y B 1$ in transgenic tobacco. $100 \mathrm{mM} \mathrm{ABA,} 50 \mathrm{mM}$ ET, and $100 \mathrm{mM}$ JA was applied to the surface of solid MS agar medium of the 3 wk-old seedlings. After $2 \mathrm{~h}$ in each treatment, sample seedlings were harvested. Relative transcript abundances of $J C R 1 M Y B 1$ in transgenic tobacco were examined using RT-qPCR. Gene-specific primers for $J c R 1 M Y B 1$ and $N t A C T$ (internal control) were used. Each point represents the mean of three replicates. Bars indicate standard errors of the mean ( \pm SEM). The Y-axis refers to the scale of the relative transcript abundance level. The X-axis denotes the WT and transgenic plants (T1, T3, and T6 lines). 
three transgenic lines is regulated by ET, ABA, and JA treatment (Figure 4D). JcR1MYBI transgenic tobacco plants affect the signaling pathways related to these three hormones.

\section{Discussion}

Compared with R2R3-type MYB TFs, reports on functional studies of single MYB-like domain TFs in plants (Du et al., 2013) are limited. Growing evidence suggests that R-MYB TFs serve vital functions in chromosomal structural maintenance and light cycle response in plants. In Arabidopsis, several R1-MYB proteins, such as EPR1 (Kuno et al., 2003), LHY (Schaffer et al., 1998), and CCA1 (Wang and Tobin, 1998), have been isolated and confirmed as circadian oscillators involved in developmental modulation in plants. In maize, R1-MYB affects developing cells in the root or shoot apex, as well as the gibberellin hormone balance (Klinge et al., 1997). The R1-MYB protein was also reported to participate in the stress response process (Cheng et al., 2013). TFs are rapidly induced during the early phases of environmental stress conditions. Functional roles for single MYB-like domain TFs, such as CCA1, have been identified in light-related and other developmental processes (Wang et al., 1997; Zhu et al., 2004). Nevertheless, other R1-type MYB TFs, such as GmMYB117 in soybean and OsMYBS3 in rice, are enhanced by abiotic and ABA stresses (Liao et al., 2008), as well as cold stress ( $\mathrm{Su}$ et al., 2010). In this study, we showed that the JcRIMYB1 transcript levels were significantly up-regulated by PEG, $\mathrm{NaCl}$, and cold treatments, and also in plants treated with $\mathrm{ABA}$ and JA. These results suggest that the single MYBlike domain TF JcR1MYB1 is involved in regulating these types of stress responses.

MYB TFs affect stress tolerance in plants. For example, AtMYB2 is induced by dehydration and ABA treatment. Over-expression of AtMYB2 results in increased sensitivity to ABA (Abe et al., 2003). AtMYB44 transgenic plants exhibit enhanced drought tolerance compared with WT plants (Jung et al., 2008). Ectopic expression of GmMYB177 confers salt and freezing tolerance in Arabidopsis (Liao et al., 2008). AtMYB41 is expressed in response to drought and salt treatment in an ABA-dependent manner. Furthermore, AtMYB41 has been shown to negatively regulate salt-induced genes, such as AtDREB2a and AtNCED3 (Lippold et al., 2009). Over-expression of StMYB1R-1 in potato plants improved plant tolerance to drought stress (Shin et al., 2011). In this work, JcR1MYB1 over-expression improved salt stress tolerance of transgenic tobacco, as demonstrated by alterations in chlorophyll content and ion leakage of the leaves in transgenic plants compared with WT. JCR1MYB1 transgenic plants also exhibited salt tolerance, as observed by comparing the seed germination rates of WT and transgenic plants in MS medium under high saline conditions. These results indicate that $J_{C} R 1 M Y B 1$ contributes to salt tolerance in physic nut plants.

\section{Acknowledgments}

This work was financially supported by the National Basic Research and Development Program (2010CB126603) and the Major Technology Project of Hainan (ZDZX2013023-1).

\section{References}

Abe H, Urao T, Ito T, Seki M, Shinozaki K and YamaguchiShinozaki K (2003) Arabidopsis AtMYC2 (bHLH) and AtMYB2 (MYB) function as transcriptional activators in abscisic acid signaling. Plant Cell 15:63-78.

Allan AC, Hellens RP and Laing WA (2008) MYB transcription factors that colour our fruit. Trends Plant Sci 13:99-102.

Chang S, Puryear J and Cairney J (1993) A simple and efficient method for isolating RNA from pine trees. Plant Mol Biol Rep 11:113-116.

Cheng L, Li X, Huang X, Ma T, Liang Y, Ma X, Peng X, Jia J, Chen S, Chen Y, et al. (2013) Overexpression of sheepgrass R1-MYB transcription factor $L c M Y B 1$ confers salt tolerance in transgenic Arabidopsis. Plant Physiol Biochem 70:252260.

Du H, Zhang L, Liu L, Tang XF, Yang WJ, Wu YM, Huang YB and Tang YX (2009) Biochemical and molecular characterization of plant MYB transcription factor family. Biochemistry (Mosc) 74:1-11.

Du H, Wang YB, Xie Y, Liang Z, Jiang SJ, Zhang SS, Huang YB and Tang YX (2013) Genome-wide identification and evolutionary and expression analyses of MYB-related genes in land plants. DNA Res 20:437-448.

Dubos C, Stracke R, Grotewold E, Weisshaar B, Martin C and Lepiniec L (2010) MYB transcription factors in Arabidopsis. Trends Plant Sci 15:573-581.

Fairless D (2007) Biofuel: The little shrub that could-maybe. Nature 449:652-655.

Jia G, Li B, Zhang D, Zhang T, Li Z, Dai J and Wang S (2009) Cloning and characterization of a novel R1-MYB transcription factor in maize. Progr Nat Sci 19:1089-1096.

Jin H and Martin C (1999) Multifunctionality and diversity within the plant MYB-gene family. Plant Mol Biol 41:577-585.

Jung C, Seo JS, Han SW, Koo YJ, Kim CH, Song SI, Nahm BH, Choi YD and Cheong JJ (2008) Overexpression of AtMYB44 enhances stomatal closure to confer abiotic stress tolerance in transgenic Arabidopsis. Plant Physiol 146:623663.

Klinge B, Lange T and Werr W (1997) The IBP gene of maize are expressed in non-meristematic, elongating cells of the seedling and in abortive floral organs. Mol Gen Genet 255:248257.

Kuno N, Moller SG, Shinomura T, Xu X, Chua HN and Furuya M (2003) The novel MYB protein EARLY-PHYTOCHROME-RESPONSIVE1 is a component of a slave circadian oscillator in Arabidopsis. Plant Cell 15:2476-2488.

Lee MM and Schiefelbein J (1999) WEREWOLF, a MYB-related protein in Arabidopsis, is a position-dependent regulator of epidermal cell patterning. Cell 99:473-483. 
Liao Y, Zou HF, Wang HW, Zhang WK, Ma B, Zhang JS and Chen SY (2008) Soybean GmMYB76, GmMYB92, and GmMYB177 genes confer stress tolerance in transgenic Arabidopsis plants. Cell Res 18:1047-1060.

Lippold F, Sanchez DH, Musialak M, Schlereth A, Scheible WR, Hincha DK and Udvardi MK (2009) AtMyb41 regulates transcriptional and metabolic responses to osmotic stress in Arabidopsis. Plant Physiol 149:1761-1772.

Openshaw K (2000) A review of Jatropha curcas: An oil plant of unfulfilled promise. Biomass Bioenergy 19:1-15.

Pontier D, Godiard L, Marco Y and Roby D (1994) hsr203J, a tobacco gene whose activation is rapid, highly localized and specific for incompatible plant/pathogen interactions. Plant J 5:507-521.

Rubio-Somoza I, Martinez M, Diaz I and Carbonero P (2006) HvMCB1, a R1MYB transcription factor from barley with antagonistic regulatory functions during seed development and germination. Plant J 45:17-30.

Sato S, Hirakawa H, Isobe S, Fukai E, Watanabe A, Kato M, Kawashima K, Minami C, Muraki A, Nakazaki N, et al. (2011) Sequence analysis of the genome of an oil-bearing tree Jatropha curcas L. DNA Res 18:65-76.

Schaffer R, Ramsay N, Samach A, Corden S, Putterill J, Carré IA and Coupland G (1998) The late elongated hypocotyls mutation of Arabidopsis disrupts circadian rhythms and the photoperiodic control of flowering. Cell 93:1219-1229.
Shin D, Moon SJ, Han S, Kim BG, Park SR, Lee SK, Yoon HJ, Lee HE, Kwon HB, Baek D, et al. (2011) Expression of StMYB1R-1, a novel potato single MYB-Like domain transcription factor, increases drought tolerance.Plant Physiol 155:421-432.

Su CF, Wang YC, Hsieh TH, Lu CA, Tseng TH and Yu SM (2010) A novel MYBS3-dependent pathway confers cold tolerance in rice. Plant Physiol 153:145-158.

Wang ZY and Tobin EM (1998) Constitutive expression of the CIRCADIAN CLOCK ASSOCIATED 1 (CCA1) gene disrupts circadian rhythms and suppresses its own expression. Cell 93:1207-1217.

Wang ZY, Kenigsbuch D, Sun L, Harel E, Ong MS and Tobin EM (1997) A Myb-related transcription factor is involved in the phytochrome regulation of an Arabidopsis Lhcb gene. Plant Cell 9:491-507.

Zhu QH, Ramm K, Shivakkumar R, Dennis ES and Upadhyaya NM (2004) The ANTHER INDEHISCENCE1 gene encoding a single MYB domain protein is involved in anther development in rice. Plant Physiol 135:1514-1525.

Associate Editor: Marcia Pinheiro Margis

License information: This is an open-access article distributed under the terms of the Creative Commons Attribution License, which permits unrestricted use, distribution, and reproduction in any medium, provided the original work is properly cited 